\title{
THE TRANSCENDENTAL LATTICE OF THE SEXTIC FERMAT SURFACE
}

\author{
Asher Auel, Christian Böhning and Hans-Christian Graf von Bothmer
}

\begin{abstract}
We prove that the integral polarized Hodge structure on the transcendental lattice of a sextic Fermat surface is decomposable. This disproves a conjecture of Kulikov related to a Hodge theoretic approach to proving the irrationality of the very general cubic fourfold.
\end{abstract}

\section{Introduction}

Proving the irrationality of a very general cubic fourfold $X \subset \mathbb{P}^{5}$ over the complex numbers is a well-known problem in algebraic geometry. At present, not a single cubic fourfold is provably irrational. However, families of rational cubic fourfolds are described by Fano [14], Tregub [32, 33], and Beauville-Donagi [6]. Hassett [18, 19] identifies, via lattice theory, a countably infinite number of subvarieties, of codimension 2 in the moduli space, consisting of rational cubic fourfolds. So far, all known rational cubic fourfolds lie on two divisors of the moduli space, corresponding to the existence of a plane or a quartic scroll marking. Even the construction of additional classes of rational cubic fourfolds is an open problem.

Recently, Kulikov [21] initiated a conjectural approach to the irrationality problem for cubic fourfolds. The strategy is modeled on that of Clemens and Griffiths [11] for cubic threefolds, with the role of the intermediate Jacobian played by the integral polarized Hodge structure $T_{X}$ on the transcendental part of the middle cohomology $H^{4}(X, \mathbb{Z})$. Assuming the existence of a birational map $r: \mathbb{P}^{4} \rightarrow X$, by Hironaka's resolution of singularities, we can resolve $r$ to a birational morphism $f: X^{\prime} \rightarrow X$ by a sequence

$$
X^{\prime}=X_{n} \rightarrow X_{n-1} \rightarrow \cdots \rightarrow X_{0}=\mathbb{P}^{4} \rightarrow X
$$

of blow-ups $X_{i} \rightarrow X_{i-1}$ along points, smooth curves, or smooth surfaces. Blow-ups along points, curves, and surfaces of $p_{g}=0$ do not contribute to the transcendental lattice, hence there is a decomposition of polarized Hodge structures

$$
T_{X^{\prime}}=\bigoplus_{j} T_{S_{j}}(-1)
$$

where the sum is taken over all surfaces $S_{j}$ of $p_{g} \geq 1$ that are the centers of blow-ups in the resolution. Here, for $S$ a smooth projective surface, $T_{S}$ denotes the transcendental part of the middle cohomology $H^{2}(S, \mathbb{Z})$. On the other hand, there is a decomposition

$$
T_{X^{\prime}}=f^{*} T_{X} \oplus\left(f^{*} T_{X}\right)^{\perp} .
$$

Received by the editors August 30, 2013.

1991 Mathematics Subject Classification. 14C30, 14D06, 14E08, 14J25, 14Q10.

Key words and phrases. Fermat surface, cubic fourfold, rationality, transcendental lattice. 
Kulikov proves [21, Lemmas 2,3] that for $X$ very general, comparing these two decompositions yields an index $j_{0}$ such that $T_{S_{j_{0}}}=f^{*} T_{X}(1) \oplus T^{\prime}$ for some nontrivial polarized Hodge substructure $T^{\prime} \subset\left(f^{*} T_{X}\right)^{\perp}$. The nontriviality of $T^{\prime}$ follows from standard estimates on the 2 nd Betti number of a minimal model of $S_{j_{0}}$. Then the irrationality of the very general cubic fourfold would follow from the following conjecture.

Conjecture 1.1 (Nondecomposability Conjecture [21, p. 59]). Let $S$ be a smooth projective surface over the complex numbers. Then the integral polarized Hodge structure $T_{S}$ on the transcendental part of $H^{2}(S, \mathbb{Z})$ is indecomposable.

For curves, the integral polarized Hodge structure on $H^{1}$ is indeed indecomposable because of Riemann's theorem describing the theta divisor of a Jacobian in geometric terms and showing that it is irreducible. Hence, the Jacobian is indecomposable as a polarized abelian variety and this is what is used substantially in the proof of the irrationality of cubic threefolds by Clemens and Griffiths [11]. An essential point in Conjecture 1.1 is the indecomposability over $\mathbb{Z}$. In fact, counterexamples over $\mathbb{Q}$ abound; note also that Jacobians of curves are decomposable if one considers them within the category of abelian varieties up to isogeny.

In this paper we prove that Conjecture 1.1 cannot be true for an arbitrary surface.

Theorem 1.2. Let $S \subset \mathbb{P}^{3}$ be the sextic Fermat surface. Then the integral polarized Hodge structure $T_{S}$ on the transcendental part of $H^{2}(S, \mathbb{Z})$ is decomposable.

After recalling some general theory in Section 2, we prove this in Section 3. Admittedly, the sextic Fermat surface $S$, defined in $\mathbb{P}^{3}$ by

$$
x_{0}^{6}+x_{1}^{6}+x_{2}^{6}+x_{3}^{6}=0
$$

is quite special. For one, it has maximal Picard $\operatorname{rank} \rho(S)=h^{1,1}(S)=86$, a fact known to Beauville, cf. [31, Remark 3.3(ii)] or [29, Ex. 3]. The rank of $T_{S}$ is 20. See also Remark 4.7 for a discussion of why having maximal Picard rank might be significant in this context.

In our analysis, we make use of the description of the integral Hodge structure of Fermat varieties as a module over the group ring of the automorphism group in the formulation of Looijenga [23, Section 2], which in turn draws on many previous sources $[17,26,28,30]$.

After a discussion of the results of this paper with the second author at an Oberwolfach workshop in May 2013, V. Kulikov suggested that his conjecture could be modified to the effect that surfaces with decomposable integral polarized Hodge structure on the transcendental lattice enjoy some Hodge theoretic rigidity property, and that this would still imply irrationality of the very general cubic fourfold. In Section 4, we work out the details of Kulikov's suggestion.

One may also wonder if surfaces $S$ for which Conjecture 1.1 fails are always defined over $\overline{\mathbb{Q}}$.

Finally, we mention a few other conjectural approaches to prove the irrationality of the very general cubic fourfold. A derived categorical approach due to Kuznetsov [22] has seen recent activity $[1,2,5,24]$. Using the theory of semiorthogonal decompositions Kuznetsov constructs a triangulated category $\mathbf{A}_{X} \subset \mathrm{D}^{b}(X)$ and conjectures 
that it encodes all the information concerning the rationality of $X$. The irrationality of the very general cubic fourfold would be a consequence. This approach runs into some difficulties due to certain pathologies that semiorthogonal decompositions of derived categories may exhibit, see $[7,8,9]$, but which can possibly be overcome if complemented by new ideas.

There is also a cohomological invariant approach due to Colliot-Thélène. Many unramified cohomology groups of $X$ vanish as a consequence of the integral Hodge conjecture [13, Theorem 1.1] proved by Voisin [35, Theorem 18] and the triviality of the Brauer group [27, Theorem A.1]. Nevertheless, by a result of Merkurjev [25, Theorem 2.11], the vanishing of all unramified cohomology groups arising from cycle modules is controlled by the vanishing of the Chow group $A_{0}\left(X_{F}\right)$ of 0 -cycles of degree 0 on $X_{F}=X \times_{\mathbb{C}} F$ over all field extensions $F / \mathbb{C}$. Hence the detection of such a nontrivial 0 -cycle on a cubic fourfold over a sufficiently complicated field $F$ would imply irrationality. Results in this direction are obtained in $[4,12,36]$.

\section{Integral polarized Hodge structures on Fermat surfaces}

In this section, we describe the integral polarized Hodge structure on the cohomology of Fermat varieties, especially Fermat surfaces, building on and developing further the description in [23]. For general background on Hodge structures, we follow [34, I Section 7.1].

\section{Definition 2.1.}

(1) An integral polarized Hodge structure (IPHS) of weight $n \in \mathbb{N}$ is a triple $\left(H_{\mathbb{Z}}, H^{p, q}, Q\right)$ where

- $H_{\mathbb{Z}}$ is a free $\mathbb{Z}$-module of finite rank and $Q: H_{\mathbb{Z}} \times H_{\mathbb{Z}} \rightarrow \mathbb{Z}$ is a nondegenerate (i.e., nondegenerate over $\mathbb{Q}$ ) bilinear form with symmetry property $Q(x, y)=(-1)^{n} Q(y, x)$ for all $x, y \in H_{\mathbb{Z}}$.

- The $H^{p, q}$, for $0 \leq p, q \leq n$, are complex linear subspaces in the complexification $H_{\mathbb{C}}=H_{\mathbb{Z}} \otimes_{\mathbb{Z}} \mathbb{C}$ with the property that

$$
H_{\mathbb{C}}=\bigoplus_{p+q=n} H^{p, q}
$$

and such that $H^{p, q}=\overline{H^{q, p}}$, with the conjugation on $H_{\mathbb{Z}} \otimes_{\mathbb{Z}} \mathbb{C}$ being induced by the conjugation on $\mathbb{C}$.

- Extend $Q$ to $H_{\mathbb{C}}$ by linearity. Then we require the orthogonality condition

$$
Q(x, y)=0 \text { if } x \in H^{p, q}, y \in H^{p^{\prime}, q^{\prime}} \text { with } p \neq q^{\prime},
$$

which is called the first Hodge-Riemann bilinear relation. We also require the positivity condition

$$
(\sqrt{-1})^{p-q} Q(x, \bar{x})>0 \text { for } 0 \neq x \in H^{p, q},
$$

which is called the second Hodge-Riemann bilinear relation, although it will not actually play any role in this paper.

(2) The notion of morphism of integral polarized Hodge structures $\left(H_{\mathbb{Z}, 1}, H_{1}^{p, q}, Q_{1}\right)$ and $\left(H_{\mathbb{Z}, 2}, H_{2}^{p, q}, Q_{2}\right)$ is the natural one: it is a $\mathbb{Z}$-linear homomorphism $f$ : $H_{\mathbb{Z}, 1} \rightarrow H_{\mathbb{Z}, 2}$ which is an isometry, i.e., $Q_{2}(f(x), f(y))=Q_{1}(x, y)$, and for 
$f_{\mathbb{C}}=f \otimes$ id we have $f_{\mathbb{C}}\left(H_{1}^{p, q}\right) \subset H_{2}^{p, q}$. Such an $f$ is necessarily an embedding since $Q_{1}$ and $Q_{2}$ are nondegenerate.

(3) There is a natural notion of direct sum of two integral polarized Hodge structures $\left(H_{\mathbb{Z}, 1}, H_{1}^{p, q}, Q_{1}\right)$ and $\left(H_{\mathbb{Z}, 2}, H_{2}^{p, q}, Q_{2}\right)$; it is simply given by

$$
\left(H_{\mathbb{Z}, 1} \oplus H_{\mathbb{Z}, 2}, H_{1}^{p, q} \oplus H_{2}^{p, q}, Q_{1} \oplus Q_{2}\right) .
$$

An integral polarized Hodge structure is indecomposable if it is not a direct sum of two nontrivial integral polarized Hodge structures.

Let $X$ be an $n$-dimensional smooth projective variety. We assume that $n=2 m$ is even. Consider the the middle cohomology $H_{\mathbb{Z}}:=H^{n}(X, \mathbb{Z}) /($ torsion $)$ and its Hodge decomposition $H_{\mathbb{C}}=H^{n}(X, C)=\bigoplus_{p+q=n} H^{p, q}$ into the spaces of harmonic $(p, q)$ forms. Consider the bilinear form $Q$ defined as the restriction to $H_{\mathbb{Z}}$ of

$$
Q(x, y)=\int_{X} x \wedge y, \quad \text { for } x, y \in H_{\mathbb{C}} .
$$

This triple almost defines an integral polarized Hodge structure; the second HodgeRiemann bilinear relation in Definition 2.1(1) is not satisfied, but it holds if we pass to primitive cohomology and replace $Q$ by $(-1)^{n+1} Q$.

Definition 2.2. Let $h \in H^{1,1} \cap H^{2}(X, \mathbb{Z})$ be a polarization class on $X$.

(a) The IPHS on the primitive middle cohomology of $X$, denoted by

$$
\left(H_{0}^{n}(X, \mathbb{Z}), H_{0}^{p, q}, Q_{0}\right)
$$

is defined as follows: $H_{0}^{n}(X, \mathbb{Z}) \subset H^{n}(X, \mathbb{Z}) /($ torsion $)$ is the kernel of the map $H^{n}(X, \mathbb{Z}) /($ tors $) \rightarrow H^{n+2}(X, \mathbb{Z}) /($ tors $)$ defined by cup product with $h$. Then $H_{0}^{p, q}=H^{p, q} \cap\left(H_{0}^{n}(X, \mathbb{Z}) \otimes_{\mathbb{Z}} \mathbb{C}\right)$ and $Q_{0}$ is the restriction of $Q$ to $H_{0}^{n}(X, \mathbb{Z})$.

(b) We call $A_{X}=H^{m, m} \cap\left(H^{n}(X, \mathbb{Z}) /(\right.$ tors $\left.)\right)$ the algebraic lattice, $A_{X}^{0}=H_{0}^{m, m} \cap$ $H_{0}^{n}(X, \mathbb{Z})$ the primitive algebraic lattice and $T_{X}=A_{X}^{\perp}$ the transcendental lattice of $X$. The transcendental IPHS is

$$
\left(T_{X}, H_{T}^{p, q}, Q_{T}\right)
$$

where $H_{T}^{p, q}=H^{p, q} \cap\left(T_{X} \otimes \mathbb{C}\right)$ and $Q_{T}$ is the restriction of $Q$ to $T_{X}$. It is an integral polarized Hodge substructure of the primitive middle cohomology.

We will now assume $n=2$ and describe this structure for the Fermat surface $X_{d}=\left\{x_{0}^{d}+x_{1}^{d}+x_{2}^{d}+x_{3}^{d}=0\right\}$ of degree $d$ in $\mathbb{P}^{3}$, taking our point of departure from [23], which we would like to simplify and amplify in several respects.

Looijenga's computation starts by considering homology. Poincaré duality gives an isomorphism

$$
P: H_{2}\left(X_{d}, \mathbb{Z}\right) \simeq H^{2}\left(X_{d}, \mathbb{Z}\right)
$$

Lemma 2.3. If we endow $H_{2}\left(X_{d}, \mathbb{Z}\right)$ with the intersection product and $H^{2}\left(X_{d}, \mathbb{Z}\right)$ with $Q$, then $P$ is an isomorphism of integral lattices. 
Proof. We have the following commutative diagram:

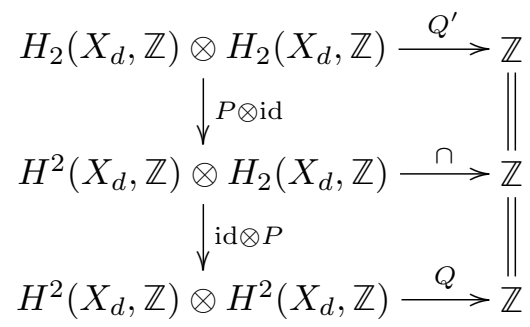

where $\cap$ is the topological cap product, $Q^{\prime}$ the intersection product, and $Q$ the bilinear form on cohomology as defined above. The assertion follows. By abuse of notation, we will also write $Q$ (and not $Q^{\prime}$ ) for the pairing on homology in the following.

Looijenga now works with the primitive homology $H_{2}^{0}\left(X_{d}, \mathbb{Z}\right)$ defined as the orthogonal to $h$ (the embedding hyperplane class from $\mathbb{P}^{3}$ ), viewed as an element of $H_{2}\left(X_{d}, \mathbb{Z}\right)$ (so this is $P^{-1}(h)$, to be precise). Hence, Poincaré duality induces an isomorphism of lattices

$$
P: H_{2}^{0}\left(X_{d}, \mathbb{Z}\right) \rightarrow H_{0}^{2}\left(X_{d}, \mathbb{Z}\right) .
$$

Remark 2.4. The Fermat hypersurface $X_{d}$ is invariant under the action of the group $\mu_{d}^{4} / \mu_{d}$ where $\mu_{d}^{4} / \mu_{d}$ acts on $X_{d}$ via rescaling the coordinates. Therefore, $H_{2}^{0}\left(X_{d}, \mathbb{Z}\right)$ and $H_{0}^{2}\left(X_{d}, \mathbb{Z}\right)$ are naturally modules over the group algebra $\mathbb{Z}\left[\mu_{d}^{4} / \mu_{d}\right]$.

The following is a consequence of [23], Corollary 2.2 and the computation following Remark 2.3 on p. 6.

Proposition 2.5. The lattice $H_{2}^{0}\left(X_{d}, \mathbb{Z}\right)$ is isomorphic, as a $\mathbb{Z}$-module, to the quotient ring

where $I_{d}$ is the ideal

$$
H_{2}^{0}\left(X_{d}, \mathbb{Z}\right) \simeq \mathbb{Z}\left[u_{0}, u_{1}, u_{2}, u_{3}\right] / I_{d}
$$

$$
I_{d}=\left(u_{0} u_{1} u_{2} u_{3}-1, \frac{u_{0}^{d}-1}{u_{0}-1}, \ldots, \frac{u_{3}^{d}-1}{u_{3}-1}\right) .
$$

The intersection form is given as follows: abbreviating

$$
u^{K}:=u_{0}^{k_{0}} \cdot \ldots \cdot u_{3}^{k_{3}} \text { for } K=\left(k_{0}, \ldots, k_{3}\right)
$$

then $u^{K} \cdot u^{L}$ is the coefficient of 1 in

$$
-u^{K-L}\left(1-u_{0}\right)\left(1-u_{1}\right)\left(1-u_{2}\right)\left(1-u_{3}\right),
$$

where we calculate in the group ring

$$
\mathbb{Z}\left[\mathrm{\mu}_{d}^{4} / \mathrm{\mu}_{d}\right]=\mathbb{Z}\left[u_{0}, u_{1}, u_{2}, u_{3}\right] /\left(u_{0} u_{1} u_{2} u_{3}-1, u_{0}^{d}-1, \ldots, u_{3}^{d}-1\right) .
$$

Moreover, the $\mathbb{Z}\left[\mathbb{\mu}_{d}^{4} / \mu_{d}\right]$-module structure on $H_{2}^{0}\left(X_{d}, \mathbb{Z}\right)$ is induced by letting the ith standard generator of $\mathbb{1}_{d}^{4}$ act via multiplication by $u_{i}$.

Remark 2.6. Let $G=\mu_{d}^{4} / \mu_{d}$. Fix a primitive $d$ th root of unity $\zeta_{d}$. The characters $\chi: G \rightarrow \mathbb{C}^{*}$ of $G$ are given by

$$
\chi\left(u_{i}\right)=\zeta_{d}^{k_{i}}, \quad k_{i} \in\{0, \ldots, d-1\} \text { and } \sum_{0 \leq i \leq 3} k_{i} \equiv 0 \bmod d .
$$


For a tuple $K=\left(k_{0}, k_{1}, k_{2}, k_{3}\right) \in\{0, \ldots, d-1\}^{4}$ with zero sum modulo $d$, we denote its associated character by $\chi_{K}$. Characters of $G$ correspond bijectively with characters of $\mathbb{Z}[G]$.

The characters of $\mathbb{Z}[G]$ that descend to characters of $H_{2}^{0}\left(X_{d}, \mathbb{Z}\right)$, via the isomorphism of Proposition 2.5 , correspond exactly to those tuples $K=\left(k_{0}, \ldots, k_{3}\right)$ with zero sum $\bmod d$ and with $k_{i} \neq 0$ for all $0 \leq i \leq 3$. Note that the complex zeros $Z$ of the ideal $I_{d}$ are precisely the points

$$
P_{K}:=\left(\zeta_{d}^{k_{0}}, \ldots, \zeta_{d}^{k_{3}}\right)
$$

with $K$ as in the preceding sentence.

We now have to describe how $H_{2}^{0}\left(X_{d}, \mathbb{Z}\right) \simeq H_{0}^{2}\left(X_{d}, \mathbb{Z}\right)$, viewed as a sublattice of $H_{0}^{2}\left(X_{d}, \mathbb{Z}\right) \otimes \mathbb{C}=H_{0}^{2}\left(X_{d}, \mathbb{C}\right)$, is positioned relative to the Hodge subspaces of $H_{0}^{2}\left(X_{d}, \mathbb{C}\right)$. Note that this will allow us to compute everything: the algebraic part, the transcendental part, and the induced integral polarized Hodge structure.

The Poincaré duality isomorphism $P$ is equivariant for the natural actions of $G$ on homology and cohomology (it is given by cap product with the fundamental class, which is invariant). Via $P$, we identify $H_{0}^{p, q} \subset H_{0}^{2}\left(X_{d}, \mathbb{C}\right)$ with its image in $H_{2}^{0}\left(X_{d}, \mathbb{C}\right)$, which we denote by the same letter. We will write $L=H_{0}^{2}\left(X_{d}, \mathbb{Z}\right)$.

Proposition 2.7. The Hodge subspace $H_{0}^{p, q} \subset L \otimes \mathbb{C}$ is

$$
H_{0}^{p, q}=\bigoplus_{\chi_{K}}(L \otimes \mathbb{C})_{\chi_{K}}
$$

where $(L \otimes \mathbb{C})_{\chi_{K}}$ is the eigenspace of the character $\chi_{K}$ and the sum runs over all characters with

$$
|K|:=\sum_{i} k_{i}=(q+1) d .
$$

In other words, for $Z^{p, q}=\left\{P_{K} \mid \sum_{i} k_{i}=(q+1) d\right\} \subset Z$,

$$
H_{0}^{p, q}=\left\{\varphi \in \mathbb{C}\left[u_{0}, \ldots, u_{3}\right] / I_{d} \mid \operatorname{supp}(\varphi) \subset Z^{p, q}\right\}
$$

Proof. We can identify the corresponding character spaces in $L \otimes_{\mathbb{Z}} \mathbb{C} \simeq H_{2}^{0}\left(X_{d}, \mathbb{C}\right)$ and $H_{0}^{2}\left(X_{d}, \mathbb{C}\right)$ via the diagram

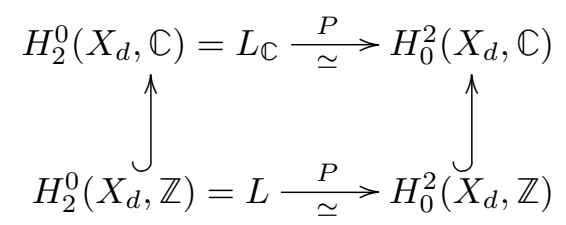

We then apply [23], section after Corollary 2.4 on p. 8 .

One can compare the character basis of Proposition 2.7 with the character basis for Griffith's presentation of the primitive Hodge subspaces in terms of residues

$$
R^{p d-4} \stackrel{\simeq}{\longrightarrow} H_{0}^{3-p, p-1}\left(X_{d}\right)
$$

where $R=\bigoplus_{j \geq 0} R^{j}$ is the Jacobian ring of $X_{d}$, see [34, II Theorem 6.10]. This comparison is made in [23], section after Corollary 2.4 . 
Lemma 2.8. The quadratic form $Q$ on $L$ can be written as

$$
Q(\varphi, \psi)=\sum_{P \in Z} \alpha_{P} \varphi(P) \psi(\bar{P})
$$

for some $\alpha_{P} \in \mathbb{Q}\left(\zeta_{d}\right)^{*}$ for each $P \in Z$.

Proof. First, note that $Q$ is invariant under $G$ and also satisfies

$$
Q(v, w)=Q(g v, g w)=\chi(g) \chi^{\prime}(g) Q(v, w),
$$

for all $v \in(L \otimes \mathbb{C})_{\chi}, w \in(L \otimes \mathbb{C})_{\chi^{\prime}}$ and all $g \in G$. Hence, $Q(v, w) \neq 0$ only if $\chi=\left(\chi^{\prime}\right)^{-1}=\overline{\chi^{\prime}}$.

Remark 2.9. The whole construction up to now is also invariant under the symmetric group $\mathfrak{S}_{4}$ acting by permutations on the $u_{i}$. Therefore, $\alpha_{P}$ is constant on the orbits of the action of $\mathfrak{S}_{4}$ on $Z$.

Proposition 2.10. Consider the action of the Galois group $\Gamma=\operatorname{Gal}\left(\mathbb{Q}\left(\zeta_{d}\right) / \mathbb{Q}\right)$ on $Z$. Let

and $Z_{T}:=Z \backslash Z_{A}$. Then

$$
Z_{A}:=\left\{P_{K} \mid \Gamma \cdot P_{K} \subset Z^{1,1}\right\}
$$

$$
A_{X}^{0}=\left\{\varphi \in \mathbb{Z}\left[u_{0}, \ldots, u_{3}\right] / I_{d} \mid \operatorname{supp}(\varphi) \subset Z_{A}\right\}
$$

and

$$
T_{X}=\left\{\varphi \in \mathbb{Z}\left[u_{0}, \ldots, u_{3}\right] / I_{d} \mid \operatorname{supp}(\varphi) \subset Z_{T}\right\} .
$$

Proof. By [30], Theorem I(iii), we get the assertion about $A_{X}^{0}$. The assertion about $T_{X}$ then follows from Lemma 2.8 .

\section{The Fermat sextic}

Let now $X_{6}$ be the sextic Fermat surface in $\mathbb{P}^{3}$ and $\zeta$ a primitive 6 th root of unity. Here, $\Gamma=\operatorname{Gal}\left(\mathbb{Q}\left(\zeta_{d}\right) / \mathbb{Q}\right)$ is generated by complex conjugation. In this case, $Z_{A}=Z^{1,1}$ and therefore by Proposition 2.10, we have that

$$
A_{X}^{0} \otimes \mathbb{C}=H_{0}^{1,1}, \quad \text { and } \quad A_{X} \otimes \mathbb{C}=H^{1,1},
$$

i.e., $X_{6}$ is a surface of maximal Picard rank. Hence

$$
T_{X} \otimes \mathbb{C}=H^{2,0} \oplus H^{0,2} .
$$

Notation 3.1. Let

$$
\begin{aligned}
& Z_{(1,1,1,3)}:=\left\{P_{(1,1,1,3)}, P_{(1,1,3,1)}, P_{(1,3,1,1)}, P_{(3,1,1,1)},\right. \\
& \left.P_{(5,5,5,3)}, P_{(5,5,3,5)}, P_{(5,3,5,5)}, P_{(3,5,5,5)}\right\}, \\
& Z_{(1,1,2,2)}:=\left\{P_{(1,1,2,2)}, P_{(2,2,1,1)}, P_{(5,5,4,4)}, P_{(4,4,5,5)}\right\} \text {, } \\
& Z_{(1,2,1,2)}:=\left\{P_{(1,2,1,2)}, P_{(2,1,2,1)}, P_{(5,4,5,4)}, P_{(4,5,4,5)}\right\} \text {, } \\
& Z_{(1,2,2,1)}:=\left\{P_{(1,2,2,1)}, P_{(2,1,1,2)}, P_{(5,4,4,5)}, P_{(4,5,5,4)}\right\}
\end{aligned}
$$

and

$$
L_{\beta}:=\left\{\varphi \in L \mid \operatorname{supp}(\varphi) \subset Z_{\beta}\right\}
$$


We have

$$
Z_{T}=Z_{(1,1,1,3)} \cup Z_{(1,1,2,2)} \cup Z_{(1,2,1,2)} \cup Z_{(1,2,2,1)}
$$

and

$$
T_{X} \otimes_{\mathbb{Z}} \mathbb{Q}=\left(L_{(1,1,1,3)} \oplus L_{(1,1,2,2)} \oplus L_{(1,2,1,2)} \oplus L_{(1,2,2,1)}\right) \otimes_{\mathbb{Z}} \mathbb{Q} .
$$

In the rest of this section we show that this decomposition holds even over $\mathbb{Z}$. The necessary computations were checked using a Macaulay2 script [3, 15].

Proposition 3.2. There is a sublattice $L_{(1,1,1,3)}^{\prime}$ of $L_{(1,1,1,3)}$ with a basis such that the intersection form is given by

$$
Q_{(1,1,1,3)}=\left(\begin{array}{cccccccc}
32 & 8 & 8 & 8 & 4 & 16 & 16 & 16 \\
8 & 32 & 8 & 8 & 16 & 4 & 16 & 16 \\
8 & 8 & 32 & 8 & 16 & 16 & 4 & 16 \\
8 & 8 & 8 & 32 & 16 & 16 & 16 & 4 \\
4 & 16 & 16 & 16 & 32 & 8 & 8 & 8 \\
16 & 4 & 16 & 16 & 8 & 32 & 8 & 8 \\
16 & 16 & 4 & 16 & 8 & 8 & 32 & 8 \\
16 & 16 & 16 & 4 & 8 & 8 & 8 & 32
\end{array}\right)
$$

We have $\operatorname{det} Q_{(1,1,1,3)}=2^{16} 3^{12}$.

Proof. Consider the matrix

$$
M_{(1,1,1,3)}=12(\zeta+1)\left(\begin{array}{llllllll}
\zeta^{4} & \zeta^{2} & \zeta^{2} & \zeta^{2} & \zeta^{1} & \zeta^{3} & \zeta^{3} & \zeta^{3} \\
\zeta^{2} & \zeta^{4} & \zeta^{2} & \zeta^{2} & \zeta^{3} & \zeta^{1} & \zeta^{3} & \zeta^{3} \\
\zeta^{2} & \zeta^{2} & \zeta^{4} & \zeta^{2} & \zeta^{3} & \zeta^{3} & \zeta^{1} & \zeta^{3} \\
\zeta^{2} & \zeta^{2} & \zeta^{2} & \zeta^{4} & \zeta^{3} & \zeta^{3} & \zeta^{3} & \zeta^{1} \\
\zeta^{1} & \zeta^{3} & \zeta^{3} & \zeta^{3} & \zeta^{4} & \zeta^{2} & \zeta^{2} & \zeta^{2} \\
\zeta^{3} & \zeta^{1} & \zeta^{3} & \zeta^{3} & \zeta^{2} & \zeta^{4} & \zeta^{2} & \zeta^{2} \\
\zeta^{3} & \zeta^{3} & \zeta^{1} & \zeta^{3} & \zeta^{2} & \zeta^{2} & \zeta^{4} & \zeta^{2} \\
\zeta^{3} & \zeta^{3} & \zeta^{3} & \zeta^{1} & \zeta^{2} & \zeta^{2} & \zeta^{2} & \zeta^{4}
\end{array}\right)
$$

Denote by $P_{i}$ the $i$ th point of $Z_{(1,1,1,3)}$. By interpolation we find polynomials $\varphi_{j}$ in $\mathbb{Z}\left[u_{0}, \ldots, u_{3}\right] / I_{6}$ with $\varphi_{j}\left(P_{i}\right)=\left(M_{(1,1,1,3)}\right)_{i j}$ and zero on all other points in $Z$. We can choose

$$
\varphi_{1}=\left(u_{3}^{4}, u_{3}^{3}, u_{3}^{2}, u_{3}, 1\right)\left(\begin{array}{c}
1 \\
s_{1}^{2}-2 s_{2}+s_{1}+2 \\
s_{1}^{2}-3 s_{2}+1 \\
-s_{1}^{2} s_{3}+3 s_{2} s_{3}-2 s_{2}^{2}-s_{3}^{2}+2 s_{1}^{2}-3 s_{2}^{2}-s_{1} s_{3}-3 s_{3}+1 \\
-2 s_{2}-1
\end{array}\right)
$$

with $s_{1}=u_{0}+u_{1}+u_{2}, s_{2}=u_{0} u_{1}+u_{0} u_{2}+u_{1} u_{2}$ and $s_{3}=u_{0} u_{1} u_{2}$. By applying appropriate permutations of the variables we obtain $\varphi_{2}, \varphi_{3}, \varphi_{4}$. The remaining polynomials are obtained from these by applying the substitution $u_{i} \mapsto u_{i}^{-1}=u_{i}^{5}$. This induces complex conjugation on the points.

Let $L_{(1,1,1,3)}^{\prime}$ be the sublattice of $L_{(1,1,1,3)}$ spanned by the $\varphi_{j}$. By Lemma 2.8 and Remark 2.9 we have that the intersection form on $L_{(1,1,1,3)}^{\prime}$ is

$$
Q_{(1,1,1,3)}=\alpha_{P_{(1,1,1,3)}} M_{(1,1,1,3)}^{t} M_{\overline{(1,1,1,3)}}
$$


where $M_{\overline{(1,1,1,3)}}$ is obtained from $M_{(1,1,1,3)}$ by interchanging the first four rows with the last four rows (since complex conjugation interchanges the first four points in $Z_{(1,1,1,3)}$ with the last four points). We compute $\alpha_{P_{(1,1,1,3)}}$ by evaluating $Q\left(\varphi_{1}, \varphi_{1}\right)$ in two different ways: firstly, by using Looijenga's formula in Proposition 2.5, and secondly, by Lemma 2.8. One finds

$$
\alpha_{P_{(1,1,1,3)}}=\frac{1}{108} .
$$

Direct computation gives the above matrix for $Q_{(1,1,1,3)}$ and its determinant.

Similarly, we have the following.

Proposition 3.3. There is a sublattice $L_{(1,1,2,2)}^{\prime}$ of $L_{(1,1,2,2)}$ with a basis such that the intersection form is given by

$$
Q_{(1,1,2,2)}=\left(\begin{array}{cccc}
24 & 12 & 0 & 0 \\
12 & 24 & 0 & 0 \\
0 & 0 & 24 & 12 \\
0 & 0 & 12 & 24
\end{array}\right)
$$

We have $\operatorname{det} Q_{(1,1,2,2)}=2^{8} 3^{6}$. The same is true for the lattices $L_{(1,2,1,2)}$ and $L_{(1,2,2,1)}$. Proof. Consider

$$
M_{(1,1,2,2)}=12(\zeta+1)\left(\begin{array}{llll}
\zeta^{0} & \zeta^{5} & \zeta^{1} & \zeta^{0} \\
\zeta^{2} & \zeta^{3} & \zeta^{4} & \zeta^{5} \\
\zeta^{5} & \zeta^{0} & \zeta^{4} & \zeta^{5} \\
\zeta^{3} & \zeta^{2} & \zeta^{1} & \zeta^{0}
\end{array}\right)
$$

Denote by $P_{i}$ the $i$ th point of $Z_{(1,1,2,2)}$. By interpolation we find polynomials $\psi_{j}$ in $\mathbb{Z}\left[u_{0}, \ldots, u_{3}\right] / I_{6}$ with $\psi_{j}\left(P_{i}\right)=\left(M_{(1,1,2,2)}\right)_{i j}$ and zero on all other points in $Z$. We can choose

$$
\begin{aligned}
\psi_{1}= & q_{1} q_{2} r_{1}^{2}-q_{1} r_{1}^{2} r_{2}+q_{1} r_{2}^{2}-q_{1}^{3} r_{1}+3 q_{1} q_{2} r_{1}+q_{2} r_{1}^{2}-q_{1}^{2} r_{2}-q_{1} r_{1} r_{2} \\
& -q_{1}^{3}+3 q_{1} q_{2}-2 q_{1}^{2} r_{1}+3 q_{2} r_{1}-q_{1} r_{2}+r_{1} r_{2}-q_{1}^{2} \\
& +2 q_{2}-q_{1} r_{1}-r_{1}^{2}+2 r_{2}-2 q_{1}-2 r_{1}-2
\end{aligned}
$$

with $q_{1}=u_{0}+u_{1}, q_{2}=u_{0} u_{1}, r_{1}=u_{2}+u_{3}$ and $r_{2}=u_{2} u_{3}$. Replacing $u_{i}$ by $u_{i}^{5}$ gives $\psi_{2}$ and the values of the second column. The third and forth columns are realized by $\psi_{3}=u_{1} \psi_{1}$ and $\psi_{4}=u_{1} \psi_{2}$. By Lemma 2.8 and Remark 2.9, we have that the intersection form on $L_{(1,1,2,2)}^{\prime}$ is

$$
Q_{(1,1,2,2)}=\alpha_{P_{(1,1,2,2)}} M_{(1,1,2,2)}^{t} M_{\overline{(1,1,2,2)}}
$$

where $M_{\overline{(1,1,2,2)}}$ is obtained from $M_{(1,1,2,2)}$ by interchanging the first two rows with the last two rows.

We compute $\alpha_{P_{(1,1,2,2)}}$ by evaluating $Q\left(\psi_{1}, \psi_{1}\right)$ in two different ways: firstly, by using Looijenga's formula in Proposition 2.5, and secondly, by Lemma 2.8. One finds

$$
\alpha_{P_{(1,1,2,2)}}=\frac{1}{72} \text {. }
$$

Direct computation gives the above matrix for $Q_{(1,1,2,2)}$ and its determinant. The existence of $L_{(1,2,1,2)}^{\prime}$ and $L_{(1,2,2,1)}^{\prime}$ with the analogous bases follows by symmetry. 
Proposition 3.4. Let

$$
T_{X}^{\prime}=L_{(1,1,3,3)}^{\prime} \oplus L_{(1,1,2,2)}^{\prime} \oplus L_{(1,2,1,2)}^{\prime} \oplus L_{(1,2,2,1)}^{\prime} .
$$

Then we have an equality of lattices $T_{X}^{\prime}=T_{X}$. In particular, always $L_{\beta}^{\prime}=L_{\beta}$.

Proof. It is clear that $T_{X}^{\prime}$ is a sublattice of $T_{X}$ of finite index. Consider the basis of $T_{X}^{\prime}$ consisting of the union of the basis vectors of the $L_{\beta}^{\prime}$ constructed above. One can check that the reductions of the vectors of this basis modulo 2 and 3 are still linearly independent. Since the discriminant of $T_{X}^{\prime}$ is only divisible by primes 2 and 3, this proves that there is no sublattice of $L$ which contains $T_{X}^{\prime}$ as a proper sublattice of finite index. In particular, $T_{X}=T_{X}^{\prime}$.

This completes the proof of Theorem 1.2.

Remark 3.5. Proposition 3.4 implies also that the discriminant of the transcendental lattice $T_{X}$ is $2^{40} 3^{30}$ and consequently the discriminant of the Picard lattice is $-2^{40} 3^{30}$ (the sign is negative since the signature of $Q$ on $H^{1,1}$ is $(1,85)$ ). We could not find this number in the literature.

Remark 3.6. We found the matrices $M_{(1,1,1,3)}$ and $M_{(1,1,2,2)}$ as follows: using Proposition 2.7 we find a $\mathbb{Q}$-basis of $L_{(1,1,1,3)}$ and of $L_{(1,1,2,2)} \oplus L_{(1,2,1,2)} \oplus L_{(1,2,2,1)}$. Clearing denominators, we find vectors in the lattice $L$ that form a basis over $\mathbb{Q}$ of $L_{(1,1,1,3)}$ and of $L_{(1,1,2,2)} \oplus L_{(1,2,1,2)} \oplus L_{(1,2,2,1)}$, respectively. These vectors generate lattices $M$ and $N$, which are not saturated, however. For each prime $p$ dividing the discriminant of $M$, for example, we reduce a set of basis vectors $\bmod p$ in the ambient $L$, and if the reductions happen to become linearly dependent, we lift the dependency relation to $\mathbb{Z}$ and find a vector divisible by $p$. Continuing in this way we arrive at a saturated sublattice $M^{\prime}$ spanning the same $\mathbb{Q}$-subspace as $M$. Using the LLL-algorithm we find vectors in $M^{\prime}$ with small coefficients. Among these we choose one with small length; evaluating this on $Z_{(1,1,1,3)}$ gives the first column of $M_{(1,1,1,3)}$. The remaining columns are obtained using the $\mathfrak{S}_{4}$-symmetry and conjugation.

\section{Rigidity and transcendental lattice decompositions}

By a (projective) family of surfaces $\pi: \mathscr{S} \rightarrow B$, we mean a flat surjective morphism of schemes or analytic spaces, all of whose fibers are projective surfaces. All families considered will actually have smooth fibers, so that $\pi$ is even a smooth map. If $B$ is a scheme or analytic space, then by a "very general" point, we mean any point outside of a suitable countable union of proper algebraic or analytic subsets.

After a discussion about Theorem 1.2, V. Kulikov suggested the following question as a possible starting point in modifying his conjecture.

Question 4.1. Let $\pi: \mathscr{S} \rightarrow B$ be a family of surfaces over an irreducible analytic space $B$ such that a very general fiber $\mathscr{S}_{b}$ has decomposable integral polarized Hodge structure on the transcendental lattice. Then must the image of the period map associated to the family necessarily be a single point?

Even while we might wonder whether Question 4.1 has a negative answer, the main result of this section is the following. 
Theorem 4.2. A positive answer to Question 4.1 would imply that the very general cubic fourfold $X$ is irrational.

The integral polarized Hodge structures on the transcendental part $T_{X}$ of the middle cohomology of a cubic fourfold $X$ are uncountably many. Moreover, in [21] it is proved that, if such $X$ were rational, their $T_{X}$ must occur as proper summands of $T_{S}$, for $S$ a smooth projective surface. Therefore, it suffices to prove the following.

Theorem 4.3. A positive answer to Question 4.1 would imply that there are only countably many (weight 2) integral polarized Hodge structures arising as a proper direct summand of the integral polarized Hodge structure on $T_{S}$ for $S$ a surface.

Remark that surfaces with $p_{g} \leq 1$ have indecomposable $T_{S}$, so one can restrict to surfaces with $p_{g} \geq 2$.

Since a given IPHS can have only countably many different decompositions, it suffices to prove that there are only countably many IPHS arising from the transcendental lattice of a surface which are decomposable.

Proof of Theorem 4.3. We divide the proof into a series of technical steps.

Step 1. There are countably many families $\mathscr{S}_{i} \rightarrow B_{i}$ of projective surfaces over irreducible base varieties $B_{i}$, such that every isomorphism class of a projective surface is represented by a fiber of some such family. One can take for example the universal families over the Hilbert schemes of two-dimensional subschemes of $\mathbb{P}^{n}, n \in \mathbb{N}$, since there are only countably many Hilbert polynomials.

Hence, it suffices to prove the following.

Lemma 4.4. Let $\pi: \mathscr{S} \rightarrow B$ be a family of surfaces over an analytic space $B$. Then the integral polarized Hodge structures arising from $T_{\mathscr{S}_{b}}$, where $\mathscr{S}_{b}$, for $b \in B(\mathbb{C})$, is a fiber and the IPHS on $T_{\mathscr{S}_{b}}$ is decomposable, are countably many.

Step 2. We first prove a linear algebra result.

Lemma 4.5. Let $D=\mathrm{SO}(2 p, q) / \mathrm{SO}(2 p) \times \mathrm{U}(q)$ be the period domain classifying polarized weight two Hodge structures on $V:=L \otimes \mathbb{C}$, where $L$ is a fixed lattice. Let $\check{D}$ be the compact dual of $D$ and $\check{D}=\operatorname{IGrass}(p, V)$, so that $D \subset \check{D}$ is a (classically) open subset.

Let $M \subset L$ be a sublattice of $L$. Let $Z \subset \check{D}$ be the subset such that $M$ is contained in the $F^{1}$-part of the Hodge filtration corresponding to the points in $Z$ (this is a closed algebraic subset). Then for each $z \in Z \cap D$, we get an induced integral polarized Hodge structure on $M^{\perp} \subset L$, by putting $H_{M^{\perp}}^{p, q}:=H^{p, q} \cap\left(M^{\perp} \otimes \mathbb{C}\right)$. Let $Z_{M} \subset$ $Z \cap D$ be the locus of points $z$ where this induced integral polarized Hodge structure is decomposable. Then $Z_{M}$ is an intersection with $D$ of countably many locally closed algebraic subvarieties of $\check{D}$.

To prove this, note first that a given lattice, $M^{\perp}$ in our case, can decompose in at most a countable number of ways into two nontrivial summands. We fix such a decomposition of $M^{\perp}$. We then have to prove that the points $z \in \check{D}$ such that the corresponding Hodge filtration decomposes in a way compatible with the fixed decomposition of $M^{\perp}$, form a finite union of locally closed algebraic subsets of $\check{D}$. This follows from the following. 
Lemma 4.6. Let $F=\operatorname{Fl}\left(k_{1}, \ldots, k_{r} ; V\right)$ be the flag variety of flags $\left(F_{1}, \ldots, F_{r}\right)$ of type $\left(k_{1}, \ldots, k_{r}\right)$ in a complex vector space $V$. Let $W \subset V$ be a fixed subspace with a given direct sum decomposition $W=W_{1} \oplus W_{2}$ into subspaces $W_{1} \subset W$ and $W_{2} \subset W$. Then the subset $Z_{W_{1}, W_{2}}$ of flags such that

$$
F_{i} \cap W=\left(F_{i} \cap W_{1}\right) \oplus\left(F_{i} \cap W_{2}\right), \quad \forall i,
$$

is a finite union of locally closed algebraic subsets.

The proof of this Lemma is straightforward: it suffices to prove it in case $F=$ $\mathrm{Fl}(k ; V)$ is a Grassmannian. The set of $k$-subspaces of $V$ intersecting $W$ in a subspace of fixed dimension $k^{\prime}$ is a locally closed subset of the Grassmannian. We now fix $k^{\prime} \leq k$ and also positive integers $a$ and $b$ with $a+b=k^{\prime}$. We have a morphism

$$
\operatorname{Gr}\left(a, W_{1}\right) \times \operatorname{Gr}\left(b, W_{2}\right) \rightarrow \operatorname{Gr}\left(k^{\prime}, W\right)
$$

(direct sum of subspaces), whose image is a closed subset $G \subset \operatorname{Gr}\left(k^{\prime}, W\right)$. Over $\operatorname{Gr}\left(k^{\prime}, W\right)$ we have the tautological bundle $\mathscr{E}$ whose fiber over a point is the given subspace of dimension $k^{\prime}$ of $W$. We consider the relative Grassmannian

$$
\psi: \operatorname{Gr}\left(k-k^{\prime}, V \otimes \mathscr{O} / \mathscr{E}\right) \rightarrow \operatorname{Gr}\left(k^{\prime}, W\right) .
$$

Now $\operatorname{Gr}\left(k-k^{\prime}, V \otimes \mathscr{O} / \mathscr{E}\right)$ is proper and has a natural morphism $f$ to $\operatorname{Gr}(k, V)$. Its image is closed (but consists also of subspaces whose dimension of intersection with $W$ is strictly larger than $k^{\prime}$ of course). In any case, $f\left(\psi^{-1}(G)\right)$, intersected with the locus of subspaces $L$ of dimension $k$ in $V$ whose intersection with $W$ has dimension exactly $k^{\prime}$, is exactly the locus of such subspaces $L$ such that $L \cap W$ decomposes into a direct sum $L \cap W_{1}$ of dimension $a$ and $L \cap W_{2}$ of dimension $b$. Since there are only finitely many choices for $a$ and $b$, the result follows.

Step 3. Now we continue with the proof of Lemma 4.4. Look at the period map

$$
p_{\mathscr{S}}: B \rightarrow D
$$

of the family $\pi: \mathscr{S} \rightarrow B$. It is holomorphic by [16]. (We may assume without loss of generality that $B$ is so small that there are no monodromy phenomena, i.e., that the local system $R^{2} \pi_{*} \mathbb{Z} \mathscr{S}$ is trivialized; certainly $B$ is covered by countably many such open subspaces). Since the Hodge filtration varies holomorphically, the locus where a given rational cohomology class of type $2 p$ remains of type $(p, p)$ is a complex analytic subspace of $B$ (even more is true, see [10], but we do not need this). It follows that the Picard rank and algebraic lattice $A_{\mathscr{S}_{b}}$ are constant (equal to $A$ ) for $b$ outside a countable union of analytic subsets of $B$, say $\left\{B_{i}\right\}_{i \in \mathbb{N}}$. Let $U=B-\bigcup_{i} B_{i}$. Look at $p^{-1}\left(Z_{A}\right) \subset B$, where $Z_{A} \subset D$ is the subset of the respective period domain from Lemma 4.5. The restriction of $\mathscr{S}$ to each irreducible component of $p^{-1}\left(Z_{A}\right)$ which meets $U$ nontrivially fulfills the hypotheses of Question 4.1. Hence, each such component gives only one isomorphism class of an integral polarized Hodge structure, assuming the a positive answer to Question 4.1. Thus the isomorphism classes of integral polarized Hodge structures coming from decomposable $T_{S}$ of fibers $\mathscr{S}_{b}$ such that $b \in U$ form a countable set. 
Step 4. We repeat the argument of Step 3 for each of the countably many families $\left.\mathscr{S}\right|_{B_{i}} \rightarrow B_{i}$. We get new analytic subsets $\left\{B_{i j}\right\}$ in each $B_{i}$ in this way (countably many) and repeat the argument for the $\left.\mathscr{S}\right|_{B_{i j}} \rightarrow B_{i j}$, and so forth. Each time, the dimension of the base decreases, and after finitely many steps, we reach zerodimensional bases (countably many). This concludes the proof of Lemma 4.4, and with it, the proof of Theorem 4.3.

Remark 4.7. We will argue that Question 4.1 has a positive answer for families of surfaces with maximal Picard rank $\pi: \mathscr{S} \rightarrow B$. Examples of such families were given by Remke Kloosterman in [20] in the form of elliptic surfaces over $\mathbb{P}^{1}$ with $p_{g}>1$ and constant $j$-invariant (these families are not isotrivial, i.e., the surfaces have moduli). This reference was kindly pointed out to us by Matthias Schütt.

To argue, we can assume that $B$ is a small, simply connected open subset of $\mathbb{C}^{n}$. If a very general fiber is a surface of maximal Picard rank, then all fibers will have maximal Picard rank. The result then follows from Proposition 4.4 in [20], or rather its proof: if $\Theta_{\mathscr{S}_{b}}$ the tangent bundle of $\mathscr{S}_{b}$ and $p$ the period map, then

$$
B \rightarrow H^{1}\left(\mathscr{S}_{b}, \Theta_{\mathscr{S}_{b}}\right) \stackrel{d_{p}}{\longrightarrow} \operatorname{Hom}\left(H^{2,0}\left(\mathscr{S}_{b}\right), H^{1,1}\left(\mathscr{S}_{b}\right)\right) \oplus \operatorname{Hom}\left(H^{1,1}\left(\mathscr{S}_{b}\right), H^{0,2}\left(\mathscr{S}_{b}\right)\right)
$$

factors over $\operatorname{Hom}\left(T^{2,0}\left(\mathscr{S}_{b}\right), T^{1,1}\left(\mathscr{S}_{b}\right)\right) \oplus \operatorname{Hom}\left(T^{1,1}\left(\mathscr{S}_{b}\right), T^{0,2}\left(\mathscr{S}_{b}\right)\right)$, which is zero because $T^{1,1}\left(\mathscr{S}_{b}\right)=0$. Therefore, $p$ is constant.

Note that the sextic Fermat surface is the only Fermat surface of maximal Picard rank; for other Fermat surfaces up to degree 8 we have not been able to decompose the IPHS on the transcendental lattices. One might wonder, therefore, whether decomposability of $T_{S}$ with its IPHS forces $S$ to have maximal Picard rank. This would certainly imply a positive answer to Question 4.1 and with it irrationality of very general cubic fourfolds.

\section{Acknowledgments}

We would like to thank A. Beauville, F. Bogomolov, L. Katzarkov, M. Schütt, Y. Tschinkel, J. Voight, and especially V. Kulikov for discussions and suggestions concerning the present material.

The first author is partially supported by a Mathematical Sciences Postdoctoral Research Fellowship grant DMS-0903039 from the NSF (US National Science Foundation) and a Young Investigator grant from the NSA. C.B is supported by HeisenbergStipendium BO 3699/1-1 of the DFG (German Research Foundation). H.-C.G.B. is supported by the RTG 1670 of the DFG (German Research Foundation).

\section{References}

[1] N. Addington and R. P. Thomas, Hodge theory and derived categories of cubic fourfolds, Duke Mathematical Journal, to appear.

[2] A. Auel, M. Bernardara, M. Bolognesi and A. Várilly-Alvarado, Cubic fourfolds containing a plane and a quintic del Pezzo surface, Algebraic Geometry, to appear.

[3] A. Auel, Chr. Böhning and H.-Chr. Graf von Bothmer, Macaulay2 scripts for "The transcendental lattice of the Fermat sextic surface", available at http://www.math.unihamburg.de/home/boehning/research/FermatSextic/M2scripts

[4] A. Auel, J.-L. Colliot-Thélène and R. Parimala, Universal unramified cohomology of cubic fourfolds containing a plane, 2013, preprint arXiv:1310.6705. 
[5] M. Bernardara, E. Macrì, S. Mehrotra and P. Stellari, A categorical invariant for cubic threefolds, Adv. Math. 229(2) (2012), 770-803.

[6] A. Beauville and R. Donagi, La variété des droites d'une hypersurface cubique de dimension 4, C.R. Acad. Sci. Paris, Série I 301 (1985), 703-706.

[7] Chr. Böhning, H.-C. Graf v. Bothmer and P. Sosna, On the derived category of the classical Godeaux surface, Advances in Mathematics, to appear.

[8] , Determinantal Barlow surfaces and phantom categories, Journal of the European Mathematical Society, to appear.

[9] — On the Jordan-Hölder property for geometric derived categories, 2012, preprint arXiv:1211.1229v1.

[10] E. Cattani, P. Deligne and A. Kaplan, On the Locus of Hodge Classes, Journal of the American Mathematical Society, 8, 483-506

[11] C. H. Clemens and P. A. Griffiths, The intermediate Jacobian of the cubic threefold, Ann. of Math. (2) 95 (1972), 281-356.

[12] J.-L. Colliot-Thélène, Descente galoisienne sur le second groupe de Chow: mise au point, 2013, preprint arXiv:1302.3215.

[13] J.-L. Colliot-Thélène and C. Voisin, Cohomologie non ramifiée et conjecture de Hodge entière, Duke Math. J. 161(5) (2012), 735-801.

[14] G. Fano, Sulle forme cubiche dello spazio a cinque dimensioni contenenti rigate rationali di quarto ordine, Comment. Math. Helv. 15 (1943), 71-80.

[15] D. R. Grayson and M. E. Stillman, Macaulay2, a software system for research in algebraic geometry, available at http://www.math.uiuc.edu/Macaulay2/

[16] Ph. Griffiths, Periods of integrals on algebraic manifolds I, II, Amer. J. Math. 90 (1968), $568-626,805-865$

[17] — On the periods of certain rational integrals I, II, Ann. Math. (2) 90 (1969), 460-495; ibid. (2) 90 (1969) 496-541

[18] B. Hassett, Special cubic fourfolds, Compos. Math. 120(1) (2000), 1-23.

[19] - Some rational cubic fourfolds, J. Algebr. Geom. 8(1) (1999), 103-114.

[20] R. Kloosterman, Extremal elliptic surfaces and infinitesimal Torelli, Michigan Math. J. 52(1) (2004), 141-161.

[21] Vik. S. Kulikov, A remark on the nonrationality problem for generic cubic fourfolds, Mat. Zametki 83(1) (2008), 61-68; Math. Notes 83(1) (2008), 57-64.

[22] A. Kuznetsov, Derived categories of cubic fourfolds, in Cohomological and geometric approaches to rationality problems, Progr. Math. 282, Birkhäuser Boston, 163-208, 2010.

[23] E. Looijenga, Fermat varieties and the periods of some hypersurfaces, Algebraic and arithmetic structures of moduli spaces (Sapporo 2007), 47-67, Adv. Stud. Pure Math. 58, Math. Soc. Japan, Tokyo, 2010.

[24] E. Macrì and P. Stellari, Fano varieties of cubic fourfolds containing a plane, Math. Ann. 354 (2012), 1147-1176.

[25] A. Merkurjev, Unramified elements in cycle modules, J. Lond. Math. Soc. (2) 78(1) (2008), 51-64.

[26] F. Pham, Formules de Picard-Lefschetz généralisées et ramification des intégrales, Bulletin de la S.M.F., tome 93 (1965), 333-367

[27] B. Poonen and J. Felipe Voloch (with appendices by J.-L. Colliot-Thélène and N.M. Katz), Random diophantine equations, Arithmetic of Higher-Dimensional Algebraic Varieties, (B. Poonen and Y. Tschinkel, eds.), pp. 175-184, Progress in Mathematics, Vol. 226, 2004, Birkhäuser Boston, Cambridge, MA.

[28] Z. Ran, Cycles on Fermat hypersurfaces, Compositio Mathematica, 42(1) (1980), 121-142.

[29] M. Schütt, Quintic surfaces with maximum and other Picard numbers, J. Math. Soc. Japan 63(4) (2011), 1187-1201.

[30] T. Shioda, The Hodge conjecture for Fermat varieties, Math. Ann. 245(2) (1979), 175-184.

[31] _ On the Picard number of a complex projective variety, Ann. Sci. École Norm. Sup. (4) 14(3) (1981), 303-321.

[32] S.L. Tregub, Three constructions of rationality of a cubic fourfold, Vestnik Moskov. Univ. Ser. I Mat. Mekh. (3) (1984), 8-14. Translation in Moscow Univ. Math. Bull. 39(3) (1984), 8-16. 
[33] _ Two remarks on four-dimensional cubics, Uspekhi Mat. Nauk. 48 (1993), no. 2(290), 201-202. Translation in Russian Math. Surveys 48(2) (1993), 206-208.

[34] C. Voisin, Hodge theory and complex algebraic geometry. I, II. Translated from the French by Leila Schneps. Reprint of the 2002 English edition. Cambridge Studies in Advanced Mathematics 76, 77, Cambridge University Press, Cambridge, 2007.

[35] _ Some aspects of the Hodge conjecture, Japan J. Math. 2(2) (2007), 261-296.

[36] Unirational threefolds with no universal codimension 2 cycle, 2013, preprint arXiv:1312.2122.

Department of Mathematics, Yale University, 10 Hillhouse Avenue, New Haven, CT 06511, USA

E-mail address: asher.auel@yale.edu

Fachbereich Mathematik der, Universität Hamburg, Bundesstrasse 55, 20146 Hamburg, GERMANY

E-mail address: christian.boehning@math.uni-hamburg.de

Fachbereich Mathematik der, Universität Hamburg, Bundesstrasse 55, 20146 Hamburg, GERMANY

E-mail address: hans.christian.v.bothmer@uni-hamburg.de 